\title{
Título de la Publicación:
}

Spatial distribution and abundance of Hemigrapsus crenulatus ( $\mathrm{H}$. Milne Edwards, 1837) (Decapoda, Varunidae) in the Puerto Cisnes estuary $\left(44^{\circ} \mathrm{S}\right.$, Aysen región, Chile)

\section{Autor:}

Vega Aguayo, Rolando; Figueroa Muñoz, Guillermo; Retamal, Marco A.; De los Rios, Patricio

\section{Datos de Publicación:}

Crustaceana, Vol.91, №12, 1465-1482, 2018

\section{Disponible en:}

https://www-scopus-com.proxybiblioteca.uct.cl/record/display.uri?eid=2-s2.0$85055627859 \&$ origin=resultslist\&sort=plf$\underline{f \& s r c=s \& s t 1=S P A T I A L+D I S T R I B U T I O N+A N D+A B U N D A N C E+O F+H E M I G R A P S U S+C R E}$ NULATUS\&st2=\&sid=583d1c7bb1eb6413b8567969efb7a827\&sot=b\&sdt=b\&sl=75 $\& \mathrm{~S}=$ TITLE-ABS-

KEY\%28SPATIAL+DISTRIBUTION+AND+ABUNDANCE+OF+HEMIGRAPSUS+CRENULA TUS\%29\&relpos $=0 \&$ citeCnt $=1 \&$ searchTerm $=$ 